\title{
Reaping the Benefits of Digitisation: Pilot study exploring revenue generation from digitised collections through technological innovation
}

\author{
Foteini Valeonti \\ University College London \\ London, UK \\ foteini.valeonti.11@ucl.ac.uk
}

\author{
Andrew Hudson-Smith \\ University College London \\ London, UK \\ a.hudson-smith@ucl.ac.uk
}

\author{
Melissa Terras \\ University of Edinburgh \\ Edinburgh, UK \\ m.terras@ed.ac.uk
}

\author{
Chrysanthi Zarkali \\ SMCA \\ Thessaloniki, Greece \\ pr@greekstatemuseum.com
}

\begin{abstract}
In the last decade significant resources have been invested for the digitisation of the collections of a large number of museums and galleries worldwide. In Europe alone, 10 million EUR is annually invested in Europeana (Europeana 2014). However, as we gradually move on from "the start-up phase" of digitisation (Hughes 2004), revenue generation and sustainability must be considered (Hughes 2004). Even beyond digitisation, generating revenue through innovation and in particular "finding new business models to sustain funding" (Simon 2011) ranks amongst museums' top challenges (Simon 2011). More importantly, despite the significant wealth of digitised assets museums now own, little has been done to investigate ways these institutions could financially benefit from their digitised collections. For art institutions in particular, this has been largely limited to the sale of image licenses, with the fear of losing this revenue posing as one of the key reasons art museums are reluctant to join the Open Content movement (Kapsalis 2016). This paper examines how recent technological advancements, such as image recognition and Print-onDemand automation, can be utilised to take advantage of the wealth of digitised artworks museums and galleries have in their possession. A pilot study of the proposed solution at the State Museum of Contemporary Art (SMCA) in Thessaloniki, Greece, is covered and the findings are examined. Early feedback indicates that there is a significant potential in the utilisation of the aforementioned technologies for the monetisation of digitised collections. However, challenges such as blending the real-world experience with the digital experience, as well as flattening the learning curve of the technological solution for museum visitors, need to be addressed. Based on the pilot study at SMCA, this paper investigates how emerging technologies can be utilised to facilitate revenue generation for all museums and galleries with digitised collections.
\end{abstract}

Museums and galleries. Digitisation. Digitised collections. Revenue generation. Mobile.

\section{INTRODUCTION}

Sustaining funding is considered one of the main challenges of museums. In November of 2017 a report initiated by the British government named budget cuts and Brexit as the two greatest challenges British museums are facing (Pes 2017). Budget cuts have been particularly severe for cultural heritage institutions in the UK, which have suffered a 69 per cent decrease in government funding since 2010 (Rodionova 2016). On an international level, arts administration expert Shapiro names fundraising one of the top three challenges of museum directors today arguing that "even well-endowed institutions like the Met find themselves needing additional financial resources to continue fulfilling their mission" (Dafoe 2017). Governments also encourage museums to become self-reliant financially (Lindqvist 2012), yet given the nature of museums, which are by definition "non-profit" institutions "in the service of society and its development" (ICOM 2007) makes it tough to pursue without deterring from their core mission. In contrast to their financial challenges however, over the last couple of decades museums have gained another arguably valuable resource, through the digitisation of their collection. Efforts to date to generate revenue from the digitised collections of museums have been largely limited to image licensing operations, which however are being increasingly questioned about their effectiveness 
and profitability, whilst attracting criticism with regards to revenue generation from public domain artworks and additionally for being a barrier for institutions interested in joining the Open Content movement. This paper introduces and discusses early results from IMS (i.e. the Infinite Museum Store); a project assisting museums generate revenue from their digitised collections. IMS implements a novel approach that utilises a mix of established (e.g. the mobile web, image recognition) and new technologies (e.g. Print-onDemand automation, Single-Page Applications). Key decisions in the design process as well as the technical details are documented below followed by an overview of early results from the first pilot of IMS at the State Museum of Contemporary Art (SMCA) that took place during the $6^{\text {th }}$ Bienalle of Contemporary Art in 2017 in Thessaloniki, Greece. This study aims to explore an alternative way for museums to generate revenue from the images of their digitised collections, whilst increasing the accessibility of their collections, offering added value to the museum visitor.

\section{BACKGROUND}

Digitisation in the cultural heritage sector can be described as the creation of digital representations of cultural and historical objects (Terras 2010), which provides numerous benefits such as "rapid access to materials held remotely" and the ability to "conserve fragile objects while presenting surrogates in more accessible forms" (Deegan \& Tanner 2002, p.32). Given digitisation's countless benefits, over the last couple of decades millions of pounds have been invested in the UK and in the whole of Europe, enabling thousands of museums, libraries and archives to digitise their collections. Centrally funded digitisation projects include UK's millennial NOF-Digitise (Nicholson 2003), JISC Content and Digitisation programme (Terras 2012) and Europeana. Investing 10 million EUR (8.9 million GBP) annually in the digitisation of Europe's cultural heritage, Europeana has already built an infrastructure that "connects more than 30 million objects from over 2,500 institutions" (Europeana 2014, p.4).

The "loss of revenue from rights and reproductions activities" ranks amongst the main "risks and downsides" for museums seeking to adopt an open access model and the only one relating to revenue generation (Kapsalis 2016, p.10), indicating that the main approach for generating revenue from digitised collections is currently through image licensing. Yet the effectiveness of this approach is increasingly being questioned. In 2004 a study titled "Reproduction charging models \& rights policy for digital images in American art museums" found that "none of the museums interviewed claimed to make any significant surplus or profits against their expenditure" (Tanner 2004, p.33). Tanner added that "everyone interviewed wants to recoup costs but almost none claimed to actually achieve or expected to achieve this", clarifying also that even those institutions that claimed to recover full costs did not account salary or overhead expenses (Tanner 2004, p.35).

There has been vocal criticism of museums charging image fees, which have been described as a "pernicious tax on scholarship", (Grosvenor 2017), suggesting "image licensing is barely profitable" for a number of UK museums (Grosvenor 2018). The National Portrait Gallery generated a profit of "just $£ 114,000$ from image fees in 2017 " which equals to $0.57 \%$ of their total yearly income (Grosvenor 2018). Another leading UK institution, the Tate generated the same year "a gross income of $£ 383,000$ from image fees", but according to Grosvenor, it also made "a startling admission" that "it doesn't know" whether the institution's image licensing operation is making a profit or a loss (Grosvenor 2018). Grosvenor has urged UK national museums to stop charging for images of works that are out of copyright (Moore 2017), because the fees "pose a serious threat to art history" (Macquisten 2017). In response, Tate explained that their licensing activities recover some of digitisation's costs and the British Museum argued that its image fees reflect the cost of making its collection, which consists of more than one million images, available online (Moore 2017).

Image license fees also pose a major barrier for museums seeking to join the Open Content movement. Adopting an open access model provides numerous benefits, ranging from the dissemination of the museum's collection to increased funding and sponsorship opportunities (Kapsalis 2016). The Rjiksmuseum, which pioneered the Open Content movement, stated that adopting this model has been highly beneficial for the institution; from gaining new sponsors (Kapsalis 2016) to greatly increasing user engagement (i.e. traffic and average time spent) on the museum's website (Terras 2015). The National Gallery of Art in Washington also stated that its Open Access policy

(https://images.nga.gov/en/page/openaccess.html) allowed the institution to "shift the emphasis" of its staff members "to helping clients and digitizing collections rather than processing paperwork", whilst increasing the public's awareness of the NGA's collections with the gallery receiving 2 million downloads in three years (Kapsalis 2016). Lastly, "a strengthened institutional brand" was also one of the benefits of open access initiatives (Kapsalis 2016, p.2). 
It is therefore necessary to explore alternative ways for generating revenue from digitised collections. Such an approach is Print-on-Demand which is described as a service that enables books, brochures and other material to be printed "when they are wanted by a customer" (Larsson 2004, p.143).

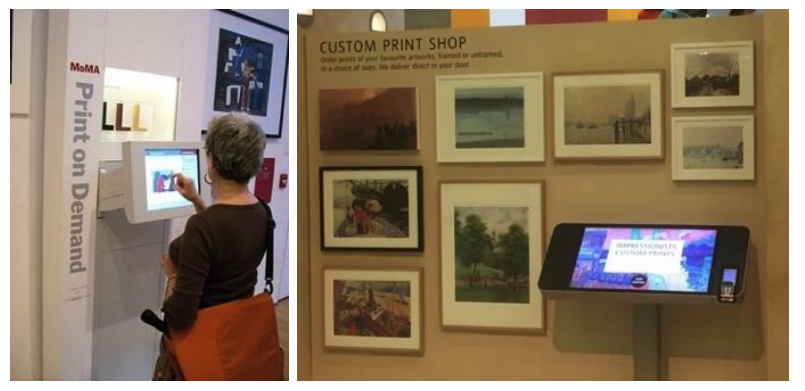

Figure 1: Print-on-Demand: MoMA in 2010 (Emmons 2010) on the left and Tate Britain in 2018 on the right.

In the case of museums and galleries, Print-onDemand refers to the purchase of custom prints and framed art prints (Figure 1). Print-on-Demand counts numerous benefits. The "primary advantage" of Print-on-Demand, as indicated by its name, is that "copies [...] are produced as they are needed", whilst another major advantage is that "no stocks of printed products need to be kept" by organisations that use Print-on-Demand (Larsson 2004, p.143). Orders are fulfilled by the Print-onDemand provider, therefore museums are not required to fulfil the orders from their own resources, as printing, packaging and shipping is undertaken by the provider, such as Kite (http://kite.ly) or Inkthreadable (http://inkthreadable.co.uk). Lastly, there are opportunities for customisation (Larsson 2004).

Given the flexibility of Print-on-Demand, freeing the institution from upfront payments, since no stock needs to be purchased or maintained, one would expect for this service to be adopted primarily from smaller museums that cannot afford to operate a large store. However, the majority of museums that currently benefit from Print-on-Demand are larger and well resourced, such as MoMA and Tate Britain (Figure 1). Looking at the way museums utilise Print-on-Demand, a typical solution is usually comprised by two components:

(i) Custom-made hardware, i.e. an installation featuring an embedded computer and a touch-screen.

(ii) Custom-made software, i.e. a software programme that runs on the computer. Linked to the museum's database, it provides a user interface that allows customers to select the artwork.
The costs of purchasing and also maintaining such a solution could arguably be prohibitive for smaller museums. Apart from operational costs, current Print-on-Demand solutions have numerous limitations; e.g. the product range is only prints and framed prints, and there is a steep learning curve for museum visitors using the custom software, which is different for each museum. More importantly, the way Print-on-Demand is adopted by museums has barely improved over the last seven years. The solution available in 2010 is the current state-of-the-art: the Print-on-Demand solution remains almost identical up to the present day (Figure 1). As a result, recent advancements in technology, such as Single Page Applications (explained below) and the maturity of established technologies, e.g. image recognition, have yet to be taken advantage of with regards to Print-onDemand solutions for museums.

\section{APPROACH}

The Infinite Museum Store (IMS), aims to enable museums to generate a revenue from their digitisation in a sustainable manner by utilising a mix of established and emerging technologies, presenting a sustainable solution that can be adopted and maintained from all museums with digitised collections, regardless of size or availability of resources such as budget, or staff time. IMS is based on Print-on-Demand for two reasons. Firstly, due to its flexibility to generate revenue, whilst freeing the institution from upfront orders and stock maintenance. Secondly, the financial potential of Print-on-Demand merchandising is significant, since the global art merchandise market is valued at 25 billion USD (Bradshaw 2011). Furthermore, to free museums from buying and maintaining custom-made hardware, IMS runs on the devices of the museum's visitor's devices (i.e. their smartphones).

Museums are encouraged to embrace their visitors' mobile devices (Petrie 2013) as apart from saving the institution from purchasing and maintaining its own hardware, the utilisation of visitors' devices has numerous other benefits. Firstly, visitors are familiar with their own smartphones and there is a flat learning curve for interaction with hardware, e.g. the camera. Secondly, their details are already stored locally, therefore it makes features such as "Login with Facebook" (utilised in our project below) simple and efficient. In addition, an increasing number of consumers use mobile internet, since this is "now [considered] the normal internet" (Zenith 2018), which is also of great advantage for museums as it frees them from the need to buy, install and maintain a visitor Wi-Fi network, provided that there is reception throughout the exhibition space. Lastly, for projects similar to IMS, 
in which the person is required to fill in their bank details in order to make a purchase as demonstrated later, the visitors' feel comfortable and secure, when using their own smartphones.

Given that the smartphones of museum visitors serve as the hardware of IMS, the software users interact with, is a mobile application. In 2013 the Metropolitan Museum of Art in New York launched a new audio-guide and the conference paper that presented an evaluation of their new audio-guide service, included the following diagram (Mann \& Tung 2015):

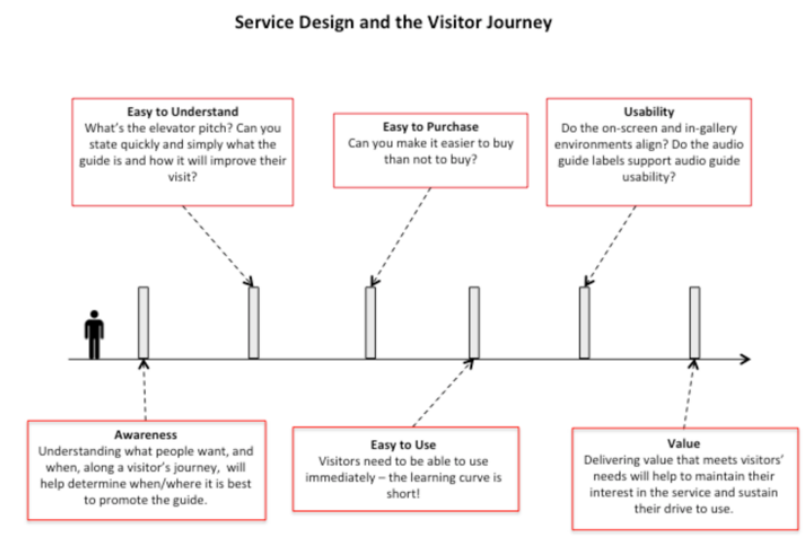

Figure 2: Barriers for using a service in the museum (Mann \& Tung 2015).

Although this diagram refers to audio-guide service design, it can be argued that it is directly applicable to all mobile applications designed for utilisation in museums. All of the diagram's barriers were individually examined when designing IMS and the measures taken to overcome each one of them are explained throughout the description of the solution.

The software of IMS is comprised by a front-end mobile application that the user interacts with and a back-end application that is hosted on the cloud (i.e. on Microsoft Azure). The front-end application is a Single-Page Application (SPA) build with Angular (http://angular.io). SPAs are web applications that "load a single HTML page and dynamically update that page as the user interacts with [them]" in order to "create fluid and responsive Web apps" (Wasson 2013). The reason this approach is adopted for the front-end application is because SPAs, by offering a fluid and responsive interface, they succeed in providing a smooth user experience similar to native applications, but without the need for users to download the application, as SPAs are web applications and can therefore be accessed using any Internet browser.

The back-end application is a cloud application developed with ASP.NET (http://asp.net). The back-end application is comprised by a series of web services that the front-end application calls asynchronously (i.e. whenever needed). There are two major services provided by the back-end application. The first one is the image recognition service, which is based on the open source Computer Vision library OpenCV (opencv.org). This service receives the photograph that is captured by the user and returns the painting it matches with. The other major service performed by the back-end is the order submission service, which receives the preferences of the user (i.e. painting, product and delivery address) and submits the order to the Print-on-Demand provider. The technical solution developed in this paper was tested as a pilot at the State Museum of Contemporary Art in Thessaloniki, Greece, during the exhibition "View from my window...Aspects of 'Home' in the Russian avant-garde. Works from the Costakis collection", which was part of the $6^{\text {th }}$ Biennale of Contemporary Art in Thessaloniki, Greece. The museum visitor experience examined next, covers both the real-world interactions, as well as the user experience of the front-end application.

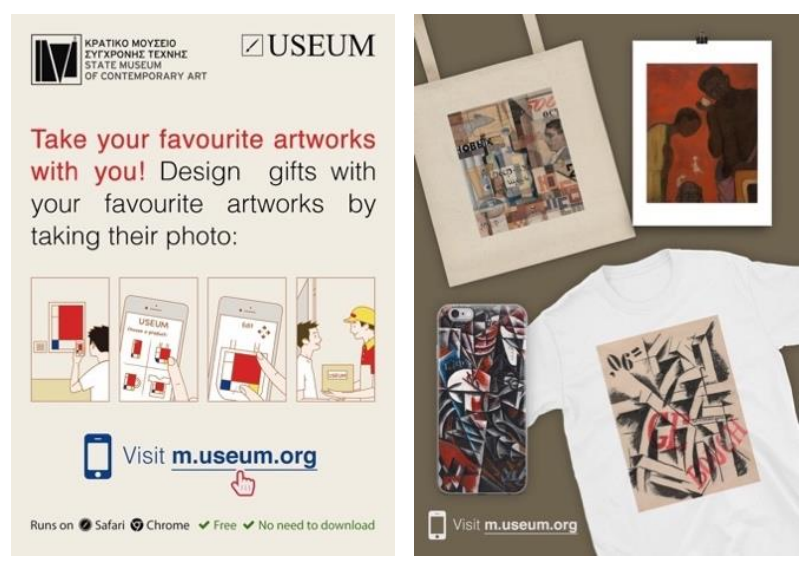

Figure 3: The flyer that is handed with the ticket.

To overcome the first barrier of the diagram in Figure 2, i.e. "Awareness", it is advised to have five points of awareness (Green 2016); in our pilot we use four such points. Three of them take place in the reception area near the ticket office. Firstly, there is a corner dedicated to the promotion of the service, comprised by a mini-stand explaining IMS and a sample product (i.e. a t-shirt featuring an artwork). Secondly, a flyer is handed to the visitors along with the ticket (Figure 3) and lastly the staff also provides a brief explanation of the service, whilst giving the flyer. The purpose of the flyer is twofold: to explain what the service offers and to inform people how they can access it (Figure 3). The last point of awareness is in the exhibition space near the artwork label, which is less detailed and serves as a reminder of the service and also as an indicator that the artwork the user is viewing can be used with IMS (Figure 8). 
When people visit the application, the first page they see, is the welcome page (Figure 4 left), which informs them about the service. The flyer along with the welcome page aim to help overcome the "Easy to Understand" barrier (Figure 2) and explain the value this application offers to visitors.
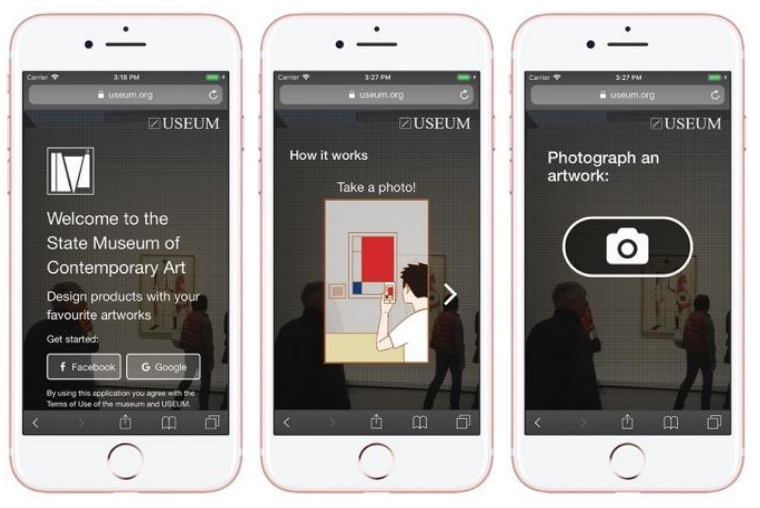

Figure 4: The front-end application of IMS.

The welcome page also prompts users to register in order to start using this application. Although registration presents an additional barrier, it is important as it allows us to stay in touch with visitors after they leave the museum. To reduce friction and increase the chances for people to successfully register, social login is utilised (i.e. registration with the use of social networks); the current implementation supports registration with Facebook and Google. Social login, in combination with the nature of the software being a web application, freeing users from downloading and installing a native application, but instead allowing them to access it from any Internet browser, aim to ensure that the application is "Easy to Purchase". IMS is a free application therefore in this case this barrier can be rephrased to "Easy to Obtain".

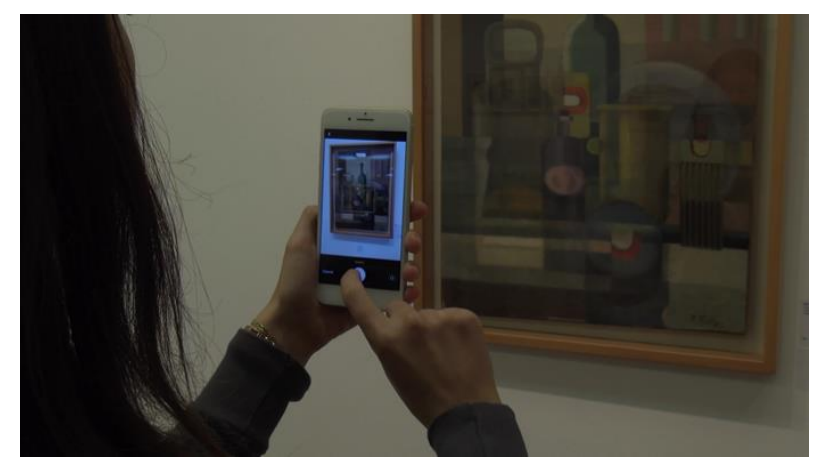

Figure 5: A museum visitor using the front-end application of IMS.

To "shorten the learning curve" as advised for overcoming the fourth barrier and ensure the application is "Easy to Use" (Figure 2), the welcome page is followed by a page titled "How it works", which presents a series of illustrations aiming to explain to users how they can use the application (Figure 4 middle). The illustrations are followed by a page prompting users to "Photograph an artwork" (Figure 4 right). For museums that prohibit photography, the application could ask users to take a photo of the artwork's label and detect the painting with an Optical Character Recognition algorithm instead. When a photograph is submitted to the application, the image recognition service of the back-end is invoked and a loading indicator is presented on screen.
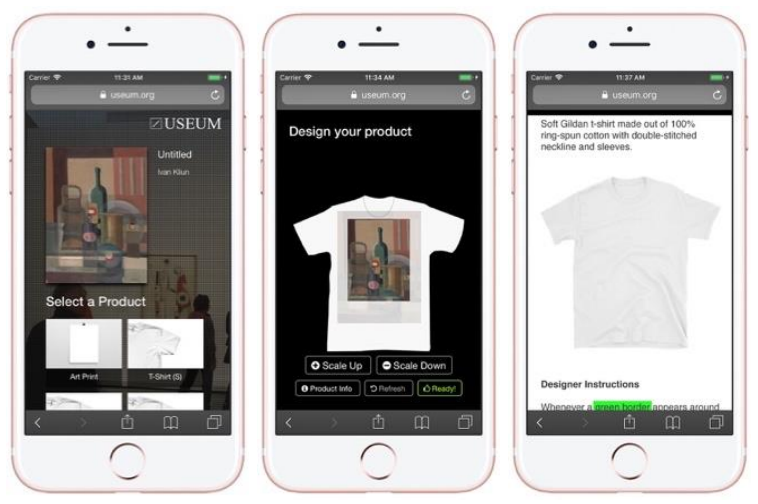

Figure 6: Pages of IMS from left to right: "Select a Product", "Design your Product" and "Product Info."

Once the user's photograph has been uploaded and the painting recognition has been completed, then users are presented with a picture of the painting that was detected, followed by a range of products for them to choose from (Figure 6 left), including art prints, t-shirts in white and in black, tote bags and iPhone cases for all models. Once a product has been selected, then the product designer is presented on screen, which allows the user to place the artwork on the product (Figure 6 middle). Users can set the exact position by dragging (i.e. tap and hold) the image on the product and also its scale by tapping on the "Scale Up" and "Scale Down" buttons. They can also restart the process by tapping on "Refresh" and read details about the selected product by tapping "Product Info" on the bottom left corner.

The product designer page, presented the greatest challenge in the design process of the front-end application. All other controls utilised, e.g. social login, or the checkout process described next, are common amongst other applications, therefore users are already familiar with them. Controls similar to the product designer page are more uncommon, subsequently a special effort was made during the design and development of this page, in order for it to be "Easy to use" (e.g. clean design, use of gestures) to overcome the fourth barrier of the diagram in Figure 2.

When users have designed their product, they can tap the button "Design is ready" on the bottom right corner to proceed (Figure 6 middle). The last two 
pages presented to the user, comprise the checkout process (Figure 7). In the first page, users are prompted to fill in the details of the delivery address the product should be (Figure 7 left), whilst the last page features the payment form (Figure 7 right).
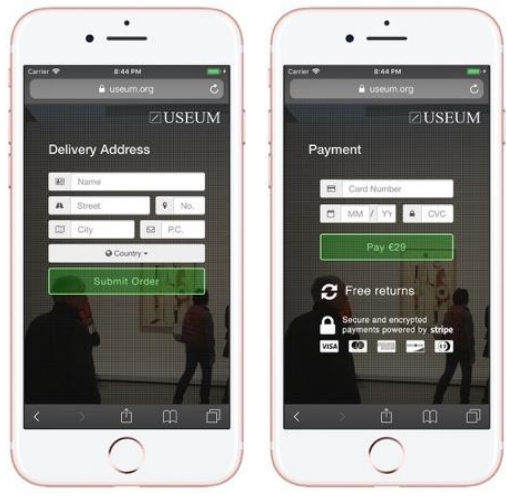

Figure 7: The checkout process of IMS

Stripe (i.e. http://stripe.com) has been used for implementing secure payments on IMS. Both the front- and back-end are integrated with the Stripe API to ensure the security of the users' payment details. When the payment has been completed and the order has been submitted successfully, a confirmation e-mail is sent to the user.

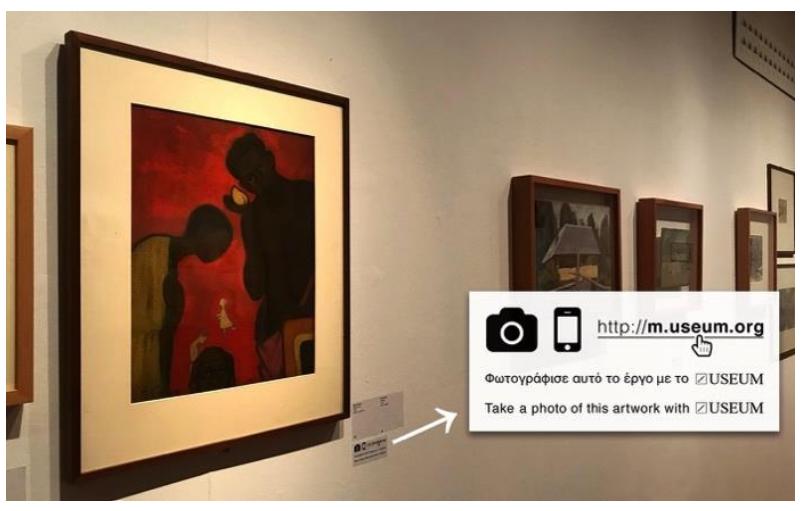

Figure 8: Label prompting visitors to capture this painting using the front-end application of IMS.

To align "the on-screen and in-gallery environments" as recommended for overcoming the sixth barrier of "Usability" (Figure 2) both environments include references to each other. The in-gallery environment features the four points of awareness, as examined previously, whilst the onscreen environment includes illustrations of how the application should be used in the exhibition space (Figure 4 middle). Lastly, the final barrier relates to "delivering value that meets the users' needs" (Figure 2). Delivering value is what determines whether those who start using the application will "maintain their interest" and the factor that will "sustain their drive to use [it]" (Figure 2). Firstly, with regards to the service, the value, i.e. what the IMS offers to visitors, is communicated as clearly as possible on the flyers, as well as on the welcome page. Sample products are available at the museum, whilst photos and details of the final products are displayed both on the flyers as well as on the "Product Info" page (Figure 6 right).

\section{EARLY RESULTS}

From approximately 300 people who received a flyer 1 in 4 (i.e. 26 per cent) registered successfully and used the application. The diagram below (Figure 9) illustrates how users progressed through the various steps from registration to checkout. The two steps that take place prior to registration (i.e. receiving the flyer and visiting the application) are not included in the diagram because accurate data for that has not been collected, as opposed to the steps that follow registration which are measured accurately.

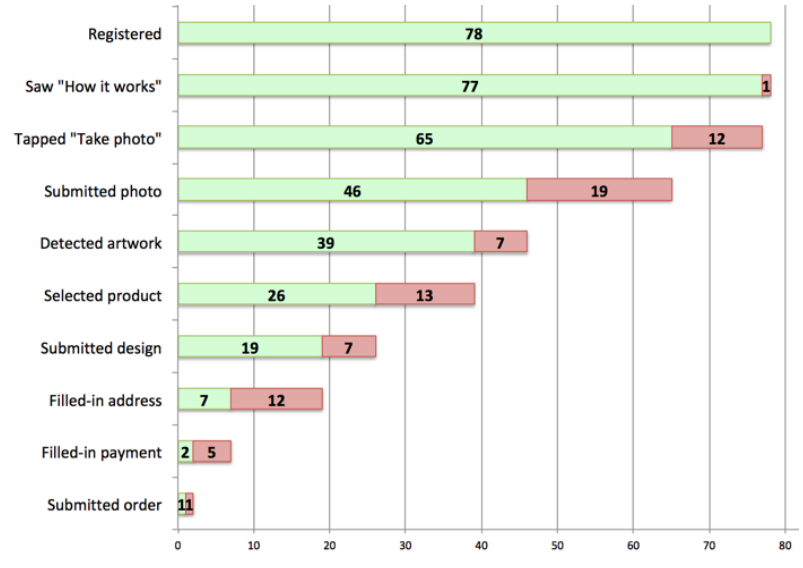

Figure 9: Usage funnel of IMS.

From the 78 people who registered, all but one, saw all 4 illustrations of the "How it works" page. From the 77 users who saw the page prompting them to capture an artwork (which appears right after the "How it works" page) 84 per cent of them tapped the camera button. Nearly 30 per cent of them did not submit a photo, whilst from the 46 users who did submit a photo 39 of them (i.e. 85 per cent) managed to successfully detect an artwork. In aggregate, from the 77 users who completed the "How it works" tutorial and saw the page prompting them to photograph an artwork (Figure 4 right) only 39 of them (i.e. 50 per cent) detected an artwork successfully. The fact 50 per cent of users did not manage to detect an artwork could be attributed to the fact that in order to successfully detect an artwork, the user must be in front of an artwork that is part of the museum's digitisation (or of another printed image of such an artwork). From the users who detected an artwork successfully, 67 per cent (i.e. 26 users) selected a product. From the 26 users who saw the product designer, 73 per cent submitted a design (i.e. 
tapped "Design is ready"). The pages of the checkout process, are the ones with the highest drop-off rate, as 63 per cent of the users, who submitted a design, did not submit their address details and 71 per cent of the users, who submitted their address details, did not submit the payment form.

Table 1: Drop-off rate for each step of the usage funnel.

\begin{tabular}{|l|c|c|}
\hline \multicolumn{1}{|c|}{ Step } & $\begin{array}{c}\text { Percentage of users } \\
\text { who proceeded from } \\
\text { previous step }\end{array}$ & $\begin{array}{c}\text { Percentage of users } \\
\text { who did not proceed } \\
\text { from previous step }\end{array}$ \\
\hline Registered & $99 \%$ & $1 \%$ \\
\hline Saw "How it works" & $84 \%$ & $16 \%$ \\
\hline Tapped "Take photo" & $71 \%$ & $29 \%$ \\
\hline Submitted photo & $85 \%$ & $15 \%$ \\
\hline Detected artwork & $67 \%$ & $33 \%$ \\
\hline Selected product & $73 \%$ & $27 \%$ \\
\hline Submitted design & $37 \%$ & $63 \%$ \\
\hline Filled-in address & $29 \%$ & $71 \%$ \\
\hline Filled-in payment & $50 \%$ & $50 \%$ \\
\hline Submitted order & & \\
\hline
\end{tabular}

From the 19 users who submitted a design, 2, i.e. 10 per cent, completed the checkout process. However, one of these 2 users used a bankcard that is not supported by our implementation and although the customer tried repeatedly to make a purchase, only one person successfully ordered a product. Another important factor is that all products, for the sake of simplicity, are priced at 29 EUR including shipping. Although that could be considered a normal price for a custom print, or a custom-designed iPhone case and t-shirt in the UK, the same products at 29 EUR in Greece can be considered expensive. In addition, visitors in Greece are not very familiar with the use of technology at the museum; indicatively only a small number of museums and cultural heritage sites provide audio-guides for their visitors. Lastly, it is also worth noting that almost half of the artworks exhibited at SMCA during the period of the study, are not supported by IMS, due to copyright restrictions. Subsequently, the fact that 10 per cent (i.e. 2 out of 19 , counting also the user who tried repeatedly to complete the order but it failed due to our implementation) of those who wanted to buy a product (i.e. had detected an artwork, selected a product and submitted a design) continued to also submit their address and payment information, it is considered highly encouraging.

\section{CONCLUSIONS AND FUTURE WORK}

Current efforts for generating revenue from a museum's digitisation focus on image licensing.
However, this approach is increasingly being questioned for its effectiveness and profitability (Tanner 2004; Grosvenor 2018), whilst also attracting criticism with regards to revenue generation from public domain works (Moore 2017). Image licensing also ranks amongst the main barriers for museums seeking to join the Open Content movement (Kapsalis 2016). Therefore, it is worth developing alternative approaches for generating revenue from digitised collections. We argue that taking advantage of Print-on-Demand via systems similar to IMS to assist museum visitors in designing their own products from digitised artworks is a particularly effective approach as it provides numerous benefits for the institution. Firstly, it allows museums to start generating revenue with no upfront financial cost: IMS is offered for free to museums, enabling access to Print-on-Demand for all organisations. Additionally, it offers added value to museum visitors by engaging them in the product creation process. Lastly, given that "accessibility" is also described as "the quality of being easy to obtain or use" (Oxford Dictionaries 2018) with a service like IMS, museums significantly increase the accessibility of their collection. In future work, we will improve the current implementation of IMS, beginning with decreasing drop-off between pages, based on the preliminary results presented above. As shown on Table 1 currently the highest drop-off occurs when users submit their address details and are presented with the payment form. We believe that integrations, such as with the popular payments platform PayPal, or with Apple Pay for iOS devices, will increase the number of people who complete the checkout process. Additionally, although IMS is currently designed to cater to art museums in particular, its concept applies to all types of museums with digitised collections. Therefore, we will explore the adaptation of IMS beyond art museums.

\section{REFERENCES}

Bradshaw, T. (2011) Internet start-up draws in art world. The Financial Times, 22 July 2011. https://www.ft.com/content/8ee39248-b485-11e0a21d-00144feabdc0 (retrieved 15 March 2018).

Dafoe, T. (2017) The Biggest Obstacles Museum Directors Face Today. Blouin Artinfo, 16 March 2017.

http://www.blouinartinfo.com/news/story/2009807/t he-biggest-obstacles-museum-directors-face-today (retrieved 15 March 2018).

Deegan, M. and Tanner, S. (2002) Digital Futures: Strategies for the information age. Neal-Schuman Publishers.

Europeana (2014) Europeana Strategy 2015-2020. https://pro.europeana.eu/files/Europeana Professio 
$\underline{\text { nal/Publications/Europeana Strategy 2020.pdf }}$ (retrieved 15 March 2018).

Green, L. (1016) What we know about mobile experiences in Museums after 6 years of research. Medium, 19 August 2016.

https://medium.com/frankly-green-webb/what-weknow-about-mobile-experiences-in-museums-after6-years-of-research-42117def2c49 (retrieved 15 March 2018).

Grosvenor, B. (2017) The reproduction fee hustle. The Art Newspaper, 20 November 2017. https://www.theartnewspaper.com/blog/thereproduction-fee-hustle (retrieved 15 March 2018). Grosvenor, B. (2018) How abolishing museum image fees could boost audiences. The Art Newspaper, 14 February 2018.

https://www.theartnewspaper.com/comment/diaryof-an-art-historian-look-beyond-the-bottom-line (retrieved 15 March 2018).

Hughes, L. M., (2004) Digitizing Collections: Strategic issues for the information manager. Facet Publishing.

ICOM (2007) Museum Definition.

http://icom.museum/the-vision/museum-definition/ (retrieved 15 March 2018).

Kapsalis, E. (2016) The Impact of Open Access on Galleries, Libraries, Museums, \& Archives. http://siarchives.si.edu/sites/default/files/pdfs/2016 0310 OpenCollections Public.pdf (retrieved 15 March 2018).

Larsson, M. (2004) The Limits of Business Development and Economic Growth. Palgrave Macmillan.

Lindqvist, K. (2012) Museum Finances: Challenges beyond Economic Crises. Museum Management and Curatorship, 27(1), pp.1-15.

Mann, L. and Tung, G. (2015) A new look at an old friend: Reevaluating the Met's audio-guide service. MW2015: Museums and the Web 2015. https://mw2015.museumsandtheweb.com/paper/anew-look-at-an-old-friend-re-evaluating-the-metsaudio-guide-service/ (retrieved 15 March 2018).

Macquisten, I. (2017) UK museums' right to charge image fees is called into question. The Art Newspaper, 28 November 2017.

https://www.theartnewspaper.com/news/museumsright-to-charge-image-fees-is-called-into-question (retrieved 15 March 2018).

Moore, M. (2017) Museum fees are killing art history, say academics. The Times, 6. November 2017. https://www.thetimes.co.uk/article/museumfees-are-killing-art-history-say-academicsqhfwmdws6 (retrieved 15 March 2018).

Nicholson, D. and Macgregor, G. (2003) "NOFDigi": putting UK culture online. OCLC Systems \& Services: International Digital Library Perspectives, 19(3), pp.96-99.
Pes, J. (2017) What Are the Biggest Challenges Facing UK Museums? Brexit and Budget Cuts, New Report Says. artnet news, 17 November 2017. https://news.artnet.com/art-world/englandsmuseums-face-brexit-on-top-of-big-budget-cutsbut-report-looks-on-bright-side-1153148 (retrieved 15 March 2018).

Oxford Dictionaries (2016) accessibility. https://en.oxforddictionaries.com/definition/accessib lity (retrieved 15 March 2018).

Petrie, M. (2013) Dear museums: the time is right to embrace mobile. The Guardian, 31 May 2013. https://www.theguardian.com/culture-professionalsnetwork/culture-professionals-

blog/2013/may/31/museums-mobile-visitors (retrieved 15 March 2018).

Rodionova, Z. (2016) UK museums to introduce entrance fees in 2016 following Government cuts. Independent, 13 January 2016.

http://www.independent.co.uk/news/business/news/ uk-museums-to-introduce-entrance-fees-in-2016following-government-cuts-a6809806.html (retrieved 15 March 2018).

Simon, N., (2011) What Are the Most Important Problems in Our Field? Museum 2.0, 3 October 2011. http://museumtwo.blogspot.fr/2011/10/whatare-most-important-problems-in-our.html (retrieved 15 March 2018).

Tanner, S. (2004) Reproduction charging models \& rights policy for digital images in American art museums: A Mellon Foundation funded study. https://kclpure.kcl.ac.uk/portal/files/48081293/USM useum SimonTanner.pdf (retrieved 15 March 2018).

Terras, M. (2010) Digital curiosities: resource creation via amateur digitization. Literary and Linguistic Computing, 25(4), pp.425-438.

Terras, M. (2012) Digitisation and Digital Resources in the Humanities. In M. Terras, C. Warwick, and J. Nyhan (eds.), Digital Humanities in Practice, pp.47-70.

Terras, M. (2015) Opening Access to collections: the making and using of open digitised cultural content. Online Information Review, 39(5), pp.733752.

Wasson, M. (2013) ASP.NET - Single-Page Applications: Build Modern, Responsive Web Apps with ASP.NET. MSDN Magazine, 28(11). https://msdn.microsoft.com/enus/magazine/dn463786.aspx (retrieved 15 March 2018).

Zenith Media (2017) Smartphone penetration to reach $66 \%$ in 2018.

https://www.zenithmedia.com/smartphonepenetration-reach-66-2018/ (retrieved 15 March 2018). 